\title{
Upregulation of GRIM-19 inhibits the growth and invasion of human breast cancer cells
}

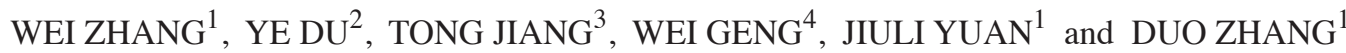 \\ Departments of ${ }^{1}$ Cosmetology Plastic Surgery, ${ }^{2}$ Breast Surgery, ${ }^{3}$ Neurological Surgery and ${ }^{4}$ Gastrointestinal Surgery, \\ The First Hospital of Jilin University, Changchun, Jilin 130021, P.R. China
}

Received July 23, 2014; Accepted April 14, 2015

DOI: $10.3892 / \mathrm{mmr} .2015 .3757$

\begin{abstract}
Gene associated with retinoid-interferon (IFN)-induced mortality 19 (GRIM-19), a novel IFN- $\beta /$ retinoic acid-inducible gene product, has been identified as a potential tumor suppressor, which is associated with the inhibition of tumor growth. GRIM-19 has been demonstrated to be downregulated in the ovarian tissue of patients with breast cancer, however, its role in breast cancer remains to be fully elucidated. In the present study, a recombinant eukaryotic expression plasmid carrying GRIM-19 was constructed and then transfected into the MCF7 human breast cancer cell line to examine its effects on breast cancer cell growth, migration and invasion using several in vitro approaches. The results demonstrated that upregulation GRIM-19 in the MCF7 cells significantly inhibited cell proliferation, colony formation, migration and invasion, and induced cell apoptosis. Additionally, upregulation of GRIM-19 also suppressed the secretion of urokinase-type plasminogen activator (u-PA), matrix metalloproteinase (MMP)-2, MMP-9 and vascular endothelial growth factor (VEGF). It was also demonstrated that the activation of signal transducer and activator of transcription 3 (STAT3) was downregulated by the expression of GRIM-19. These results revealed that overexpression of the GRIM-19 gene may be an effective approach to control the growth and invasion of human breast cancer cells.
\end{abstract}

\section{Introduction}

Breast cancer is the most common type of malignancy in females and is a leading cause of cancer-associated mortality among females worldwide (1). In the United States, it was estimated that $\sim 230,000$ females were likely to be diagnosed with breast cancer, with an associated mortality rate of $>40,000$ in 2013 (2). Surgery and chemotherapy are increasingly used to

Correspondence to: Professor Duo Zhang, Department of Cosmetology Plastic Surgery, The First Hospital of Jilin University, 71 Xinmin Street, Changchun, Jilin 130021, P.R. China

E-mail: zhangduo723@163.com

Key words: breast cancer, GRIM-19, STAT3, invasion treat $\mathrm{BC}$, however, the outcome remains poor due to the lack of specific early symptoms, effective tumor biomarkers and effective treatments (3). In addition, breast cancer is known to be complex and heterogeneous in its development, progress and response to treatment (4). Accordingly, identifying the molecular mechanisms underlying breast cancer carcinogenesis is likely to improve current understanding of the pathogenesis of breast cancer and, ultimately, lead to the development of sensitive and specific molecular markers and novel therapies.

Gene associated with retinoid-interferon (IFN)-induced mortality 19 (GRIM-19) was originally isolated and identified as a growth suppressive gene product involved in IFN- $\beta$ - and retinoic acid-induced cell death in mammary carcinoma cells (5). GRIM-19, a member of the GRIM family, is located on human chromosome 19p13.1 and encodes a novel $16 \mathrm{kDa}$ protein, which is present in the cytoplasm, nucleus and mitochondria of cells $(6,7)$. Previous studies have demonstrated that loss of the expression of GRIM-19 occurs in several human carcinomas, including liver (8), kidney (9), cervical (10), lung (11) and laryngeal cancer (12), and mutations in the GRIM-19 gene have been identified in thyroid tumors $(13,14)$ and in head and neck cancer $(15)$. In addition, overexpression of GRIM-19 has been observed to inhibit cell proliferation, migration and invasion, and to induce apoptosis in human prostate, liver and gastric cancer, and in renal carcinoma cells (16-20). In breast cancer, despite a previous study demonstrating that the downregulation of GRIM-19 correlates with aggressive clinicopathologic features in breast cancer, including lymph node metastases, and thus an advanced tumor, node, metastasis stage (21), its detailed role in the proliferation, apoptosis, migration and invasion of breast cancer cells remains to be fully elucidated.

The present study aimed to investigate the effects of GRIM-19 on the proliferation, apoptosis, migration and invasion in breast cancer cells, by transfecting cells with a plasmid constructed to overexpress GRIM-19. The findings of this investigation may contribute to the development of gene therapy for breast cancer, by using GRIM-19 as a target.

\section{Materials and methods}

Cell lines and cell culture. The MCF-7 human breast cancer cell line was purchased from the Cell Bank of Type Culture Collection of the Chinese Academy of Sciences, Shanghai 
Institute of Cell Biology, Chinese Academy of Sciences, (Shanghai, China) and was cultured in Dulbecco's modified Eagle's medium (Gibco Life Technologies, Gaithersburg, MD, USA), supplemented with $10 \%$ fetal bovine serum (Gibco Life Technologies), $100 \mathrm{U} / \mathrm{ml}$ penicillin (Sigma-Aldrich, St. Louis, MO, USA) and $0.1 \mathrm{mg} / \mathrm{ml}$ streptomycin (Sigma-Aldrich), in a humidified atmosphere of $5 \% \mathrm{CO}_{2}$ and $37^{\circ} \mathrm{C}$.

Plasmid construction and transfection. A full-length open reading frame of the human GRIM-19 gene was cloned from the MCF-7 cells using polymerase chain reaction (PCR). The primers (Takara Biotechnology Co., Inc., Dalian, China) for GRIM-19 cloning were as follows: Sense, 5'-GAGAATTCATGGCGGCGTCAAAGG-3' (EcoRI) and antisense 5'-GACTCGAGCAGGGCCTACGTGTACCACAT-3' (XhoI). The cDNA of GRIM-19 was cloned into the XhoI and EcoRI sites of the pcDNA3.1 mammalian expression vector (Invitrogen Life Technologies, Carlsbad, CA, USA) and was termed pGRIM-19. The successful construction of the recombinant plasmid, pGRIM-19, was confirmed by DNA sequencing (BIG, Guangzhou, China). pGRIM-19 was transfected into the MCF-7 cells using Lipofectamine 2000 (Invitrogen Life Technologies), according to the manufacturer's instructions. Following $72 \mathrm{~h}$ culture at $37^{\circ} \mathrm{C}$, the cells were harvested to determine the mRNA and protein expression levels of GRIM19 using RT-qPCR and western blotting, respectively.

Reverse transcription-quantitative PCR (RT-qPCR). The mRNA expression levels of signal transducer and activator of transcription 3 (STAT3) and GRIM-19 were assessed using RT-qPCR. The cells, which were transfected with the pGRIM-19 plasmids were collected by centrifugation at $1,000 \mathrm{x} \mathrm{g}$ for $5 \mathrm{~min}$ at room temperature $72 \mathrm{~h}$ after transfection. The total RNA was extracted using TRIzol reagent (Invitrogen Life Technologies). The first strand cDNA was reverse transcribed with $2 \mu \mathrm{g}$ total RNA, using a Takara Reverse Transcription kit (Takara Biotechnology Co., Inc.) and oligo (dT) 15 primers (Takara Biotechnology Co., Inc.). Amplification of GRIM-19 was performed with 1 cycle at $95^{\circ} \mathrm{C}$ for $5 \mathrm{~min}$, and 30 cycles of $95^{\circ} \mathrm{C}$ for $15 \mathrm{sec}, 55^{\circ} \mathrm{C}$ for $30 \mathrm{sec}$ and $72^{\circ} \mathrm{C}$ for $1 \mathrm{~min}$, with a final extension for $5 \mathrm{~min}$ The primers used for STAT3 and GAPDH were as follows: STAT3, sense 5'-GAGTCAGGCACTGTGGG-3' and antisense 5'-CGGTCGGTTTCTGCCTGTA-3' and GAPDH, sense 5'-CCTTCATTGACCTCAACTA-3' and antisense 5'-GGAAGGCCATGCCAGTGAGC-3'. GAPDH was used as a control. The qPCR reactions were performed on an ABI 7900 Fast system (Applied Biosystems, Foster City, CA, USA) as follows: Initial denaturation at $94^{\circ} \mathrm{C}$ for $5 \mathrm{~min}$, followed by 30 cycles of amplification $\left(94^{\circ} \mathrm{C}\right.$ for $30 \mathrm{sec}, 58^{\circ} \mathrm{C}$ for $45 \mathrm{sec}$ and $72^{\circ} \mathrm{C}$ for $90 \mathrm{sec}$ ) and a final extension of $10 \mathrm{~min}$ at $72^{\circ} \mathrm{C}$. The reaction products were analyzed using $1.5 \%$ standard agarose gel electrophoresis (Sigma-Aldrich) and visualized using a GIS Gelatumimaging system (Tanon, Shanghai, China).

Western blot analysis. The culture supernatants were collected by centrifugation at $1,000 \mathrm{x}$ g for $5 \mathrm{~min}$ at room temperature and concentrated $\sim 20$-fold using a spin-concentrator (Millipore, Bedford, MA, USA) $72 \mathrm{~h}$ after transfection. The proteins from the cell supernatants and the lysates were measured using a bicinchoninic acid (Sigma-Aldrich) method. Equal quantities of protein $(30 \mu \mathrm{g})$ were loaded into each lane of 10-15\% SDS-PAGE gels (Sigma-Aldrich) and subsequently transferred onto polyvinylidene difluoride membranes (Sigma-Aldrich). The membranes were blocked with $5 \%$ non-fat milk to inhibit non-specific binding, and the membranes were then incubated overnight at $4^{\circ} \mathrm{C}$ with the following antibodies: Rabbit polyclonal anti-human Survivin (1:5,000; cat. no. 2803S; Cell Signaling Technology, Inc., Danvers, MA, USA), mouse monoclonal anti-human B-cell lymphoma (BCL)-2 (1:3,000; cat. no. sc-7382; Santa Cruz Biotechnology, Inc., Santa Cruz, CA, USA), rabbit polyclonal anti-human matrix metalloproteinase (MMP)-2 (1:1,000; cat. no. 4022S; Cell Signaling Technology, Inc.), mouse monoclonal anti-human MMP-9 (1:2,000; cat. no. sc-12759; Santa Cruz Biotechnology, Inc.), rabbit polyclonal anti-human urokinase-type plasminogen activator (u-PA; 1:2,000; cat. no. sc-14019; Santa Cruz Biotechnology, Inc.), mouse monoclonal anti-human GRIM-19 (1:1,000; cat. no. sc-365987; Santa Crüz Biotechnology, Inc.) and mouse monoclonal anti-human Stat3 (1.2,000; cat. no. sc-8019; Santa Cruz Biotechnology, Inc.). Mouse monoclonal anti-human GAPDH (1:10,000; cat. no. sc-365062; Santa Cruz Biotechnology, Inc.) was used as a loading control. The membranes were then incubated with polyclonal goat anti-mouse horseradish peroxidase-conjugated immunogloblin G (1:10,000; cat. no. sc-2005; Santa Cruz Biotechnology, Inc.) or polyclonal goat anti-rabbit horseradish peroxidase-conjugated immunoglonulin $\mathrm{G}$ (1:10,000; cat. no. sc-2004; Santa Cruz Biotechnology, Inc.) for $2 \mathrm{~h}$ at room temperature and the protein bands were visualized using a supersignal enhanced chemiluminescence detection kit (Pierce, Rockford, IL, USA).

Cell proliferation assay. Cell proliferation was assessed using an MTT cell proliferation kit (Roche Applied Science, Indianapolis, IN, USA), according to the manufacturer's instructions. Briefly, the cells $\left(2 \times 10^{3} /\right.$ well $)$ were seeded into a 96 -well plate and incubated for $2 \mathrm{~h}$ at $37^{\circ} \mathrm{C}$. Cell growth was measured using color generation (MTT reduction) by adding $20 \mu \mathrm{l}$ MTT $(5 \mathrm{mg} / \mathrm{ml})$ to the culture medium. The cells were harvested and incubated with $100 \mu \mathrm{l}$ dimethyl sulfoxide (Sigma-Aldrich) for $30 \mathrm{~min}$, and absorbance was measured using an enzyme-linked immunosorbent assay (ELISA) multi-well spectrophotometer (SpectraMax M3; Molecular Devices Corp., Sunnyvale, CA, USA) at a $490 \mathrm{~nm}$ test wavelength. All assays were performed in triplicate and data are expressed as the mean \pm standard deviation.

Colony formation assay. The cells were seeded at a density of 1,000 cells/well in 24-well tissue culture plates. The plates were incubated for 2 weeks in a humidified incubator at $37^{\circ} \mathrm{C}$. After 3 weeks, the colonies were stained using $0.05 \%$ crystal violet (Sigma-Aldrich), containing 50\% methanol (Sigma-Aldrich). The colonies were counted in between four and five randomly selected fields for each of the duplicate samples using a microscope (IX51; Olympus, Tokyo, Japan) at magnification, x100.

Cell apoptosis. For the analysis of apoptosis, the MCF-7 cells were collected by centrifugation at $1,000 \mathrm{x} \mathrm{g}$ for $5 \mathrm{~min}$ at room temperature $48 \mathrm{~h}$ after transfection, diluted to a 
concentration of $1 \times 10^{6}$ cells $/ \mathrm{ml}$ and washed three times with ice cold phosphate buffered saline (PBS; Sigma-Aldrich). The cells were then incubated with phycoerythrin (PE) annexin-V and 7AAD using a PE Annexin V Apoptosis Detection kit I (BD Pharmingen, San Diago, CA, USA), according to the manufacturer's instructions. The cells were then analyzed using fluorescence-activated cell sorting (FACS Calibur; BD Pharmingen, San Diago, CA, USA). The experiments were performed in triplicate. In addition, the expression levels of survivin and Bcl-2 were also assessed using western blot analysis as an additional indicator of apoptosis.

Wound-healing assay. A wound-healing assay was performed to assess the effect of GRIM-19 on cell migration. Briefly, the cells $\left(2 \times 10^{4}\right)$ were cultured to confluency and transfected with the pGRIM-19 plasmid $(1 \mu \mathrm{g})$ for $4 \mathrm{~h}$ at $37^{\circ} \mathrm{C}$ to arrest cell proliferation. A wound track was made using a $200 \mu \mathrm{l}$ pipette tip and the medium and any cell debris were removed. The plates were washed with PBS and the cells were grown in fresh medium for a further $24 \mathrm{~h}$. The cells invading the wound line were observed under an inverted phase-contrast microscope (Leica DMR; Leica, Wetzlar, Germany).

Invasion assay. Cell invasion was measured using a Matrigel-coated film insert ( $8 \mu \mathrm{m}$ pore size) in 24 -well invasion chambers (BD Biosciences). The cells $\left(5 \times 10^{4}\right)$, which were suspended in $200 \mu 1$ FBS-free DMEM, were added to the upper compartment of the invasion chamber and $500 \mu 1$ complete media was added to the lower chamber. Following incubation for $24 \mathrm{~h}$, the cells on the upper side of the filter were removed and the cells in the lower surface of the filter were stained with hematoxylin and eosin (Sigma-Aldrich). The cell number was counted using a microscope (Olympus, Tokyo, Japan) at a magnification of $x 200$. The mean values were calculated by averaging the total numbers of cells from ten randomly selected microscopic fields. The assay was performed in triplicate.

Measurement of VEGF production. VEGF production was determined using a competitive ELISA. Briefly, the MCF7 cells $\left(2 \times 10^{4}\right)$ were transfected with the pGRIM-19 plasmid in 12 -well plates for $24 \mathrm{~h}$ at $37^{\circ} \mathrm{C}$. The culture media was subsequently centrifuged to remove the cell debris. The cell-free culture media was collected by centrifugation at $1,000 \mathrm{x} \mathrm{g}$ for $5 \mathrm{~min}$ at room temperature at indicated time-points and the levels of VEGF were measured using a Human VEGF ELISA kit (Yanyu, Shanghai, China), according to the manufacturer's instructions.

Statistical analysis. All experiments were performed at least three times independently and the data are expressed as the mean \pm standard deviation. $\mathrm{P}<0.05$ was considered to indicate a statistically significant difference. All statistical analyses were performed using SPSS software (version 19.0; SPSS Inc., Chicago, IL, USA) and GraphPad Prism version 5.01 (GraphPad Software, San Diego, CA, USA) for Windows ${ }^{\circledR}$.

\section{Results}

pGRIM-19 affects the expression of GRIM-19 in breast cancer cells. GRIM-19 was amplified using PCR and subcloned into
A

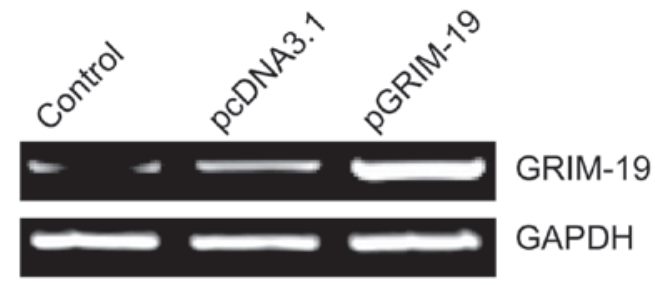

B
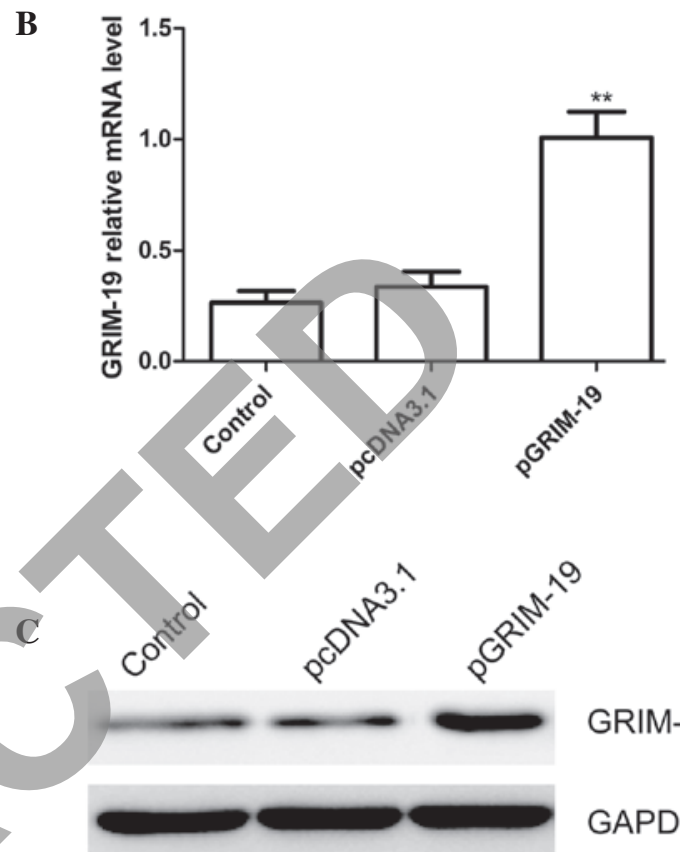

GRIM-19

GAPDH

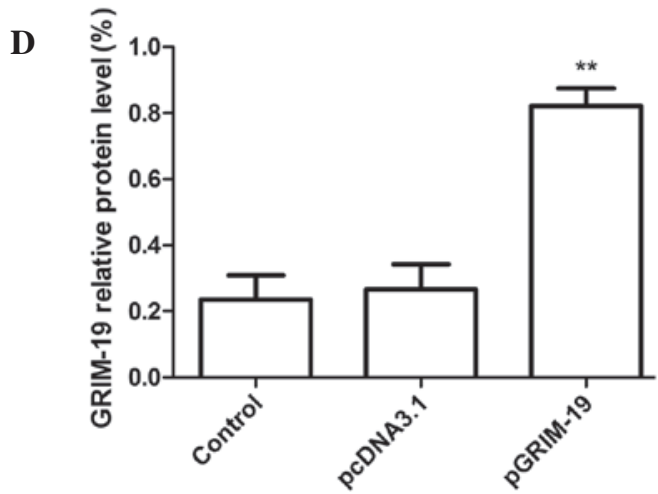

Figure 1. Transfection with pGRIM-19 increases the expression of GRIM-19 in MCF7 cells. (A) Reverse transcription-quantitative polymerase chain reaction analysis of the mRNA expression of GRIM-19 $72 \mathrm{~h}$ after plasmid transfection. (B) Relative quantification of the mRNA levels of GRIM-19 using densitometric analysis. (C) Western blot analysis of the protein expression of GRIM-19 $72 \mathrm{~h}$ after transfection. GAPDH was used as an internal control. (D) Relative quantification of the protein levels of GRIM-19 using densitometric analysis. Data are expressed as the mean \pm standard deviation. ${ }^{* *} \mathrm{P}<0.01$, vs. control. GRIM-19, gene associated with retinoid-interferon-induced mortality 19 VEGF, vascular endothelial growth factor; pGRIM-19, GRIM-19-overexpression plasmid; pcDNA3.1, blank plasmid.

the pcDNA3.1 vector. The resulting plasmid, pGRIM-19, was them confirmed by sequence analysis. The results of sequence alignment demonstrated that the GRIM19 gene shared a sequence homology of $100 \%$ with the GRIM-19 published 
A

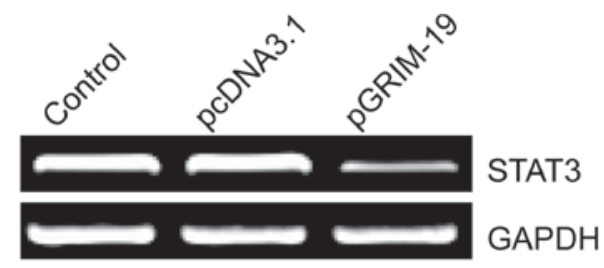

B

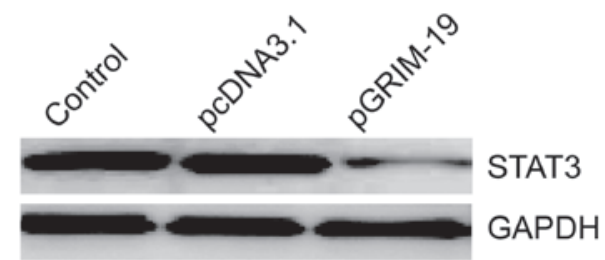

Figure 2. Upregulation of the expression of GRIM-19 inhibits the expression of STAT3 in MCF-7 cells. (A) Reverse transcription-quantitative polymerase chain reaction analysis of the mRNA expression of STAT3 $72 \mathrm{~h}$ after plasmid transfection. (B) Western blot analysis of the protein expression of STAT3 using specific antibodies against STAT3 following treatment with the indicated plasmid. GAPDH was used as an internal control. GRIM-19, gene associated with retinoid-interferon-induced mortality 19; STAT, signal transducer and activator of transcription; pGRIM-19, GRIM-19-overexpression plasmid; pcDNA3.1, blank plasmid.

in GeneBank (no. AF155662). The pGRIM-19 or pcDNA3.1 blank plasmid were transfected into the MCF7 cells, and the mRNA and protein expression levels of GRIM-19 were determined using RT-qPCR and western blot analyses, respectively. The results of the RT-qPCR revealed that the expression of GRIM-19 was significantly increased in the MCF-7 cells transfected with the pGRIM-19 plasmid, compared with the untreated cells and the cells transfected with the blank vector $(\mathrm{P}<0.05$; Fig. 1A and B). The protein expression of GRIM-19 was also significantly upregulated following transfection with the pGRIM-19 plasmid $(\mathrm{P}<0.05 ;$ Fig. $1 \mathrm{C}$ and $\mathrm{D})$.

Upregulation of GRIM-19 inhibits the expression of STAT3. To evaluate the association between GRIM-19 and its target gene, STAT3, in breast cancer cells, the present study examined the effects of upregulated GRIM-19 on the expression of STAT3 in breast cancer cells. As shown in Fig. 2A, increased levels of GRIM-19 in the MCF-7 cells resulted in a marked decrease in the mRNA expression of STAT3 at $72 \mathrm{~h}$ after transfection (Fig. 2A). Similar results were confirmed in the western blot analysis (Fig. 2B).

Upregulation of GRIM-19 inhibits cell proliferation and colony formation in MCF7 cells. To investigate whether the upregulation of GRIM-19 affects cell proliferation, an MTT assay was performed. The results revealed that increased levels of GRIM-19 in the MCF-7 cells resulted in a notable decrease in cell proliferation $(\mathrm{P}<0.01$; Fig. $3 \mathrm{~A})$.

In addition, the effects of the upregulation of GRIM-19 on breast cancer cell colony formation ability were assessed. As shown in Fig. 3B, overexpression of GRIM-19 in the MCF-7 cells reduced the colony number, compared with those observed in the blank vector or control groups $(\mathrm{P}<0.05)$.

Upregulation of GRIM-19 induces apoptosis in MCF-7 cells. To investigate whether upregulation of GRIM-19 induced cell
A
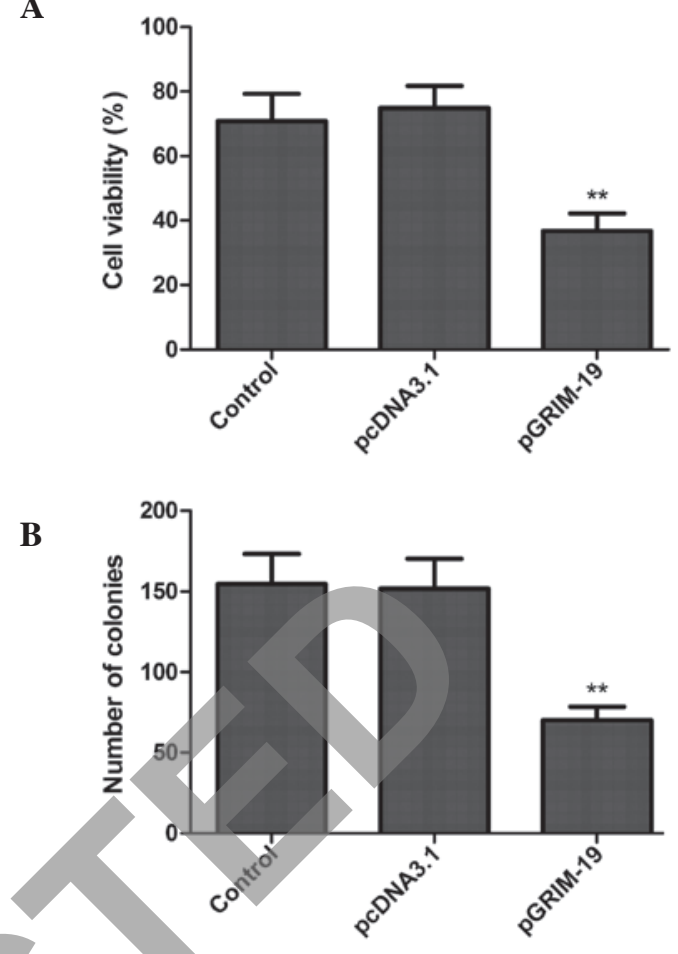

Figure 3. Upregulation of the expression of GRIM-19 inhibits cell proliferation and cell colony formation in MCF-7 cells. (A) Cell proliferation was determined using an MTT assay. (B) Cell colony formation was evaluated following plasmid transduction. Data are expressed as the mean + standard deviation. ${ }^{* * *} \mathrm{P}<0.01$, vs. control. GRIM-19, gene associated with retinoid-interferon-induced mortality 19; pGRIM-19. GRIM-19-overexpression plasmid pcDNA3.1, blank plasmid.

A

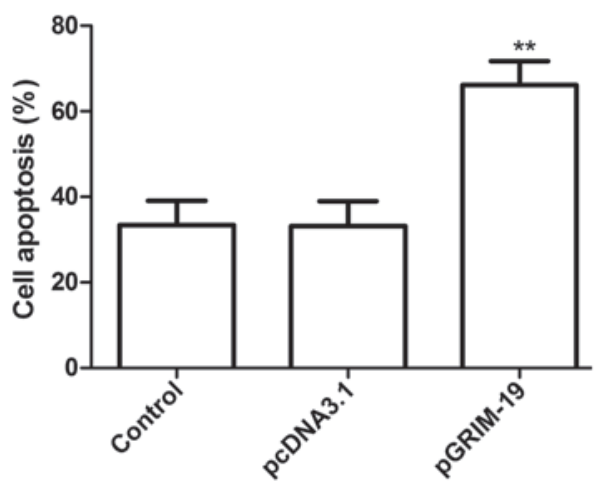

B

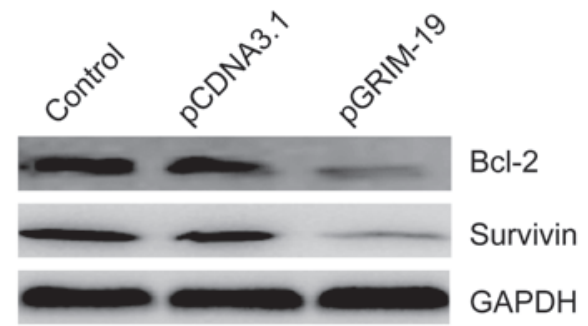

Figure 4. Upregulation of the expression of GRIM-19 induces apoptosis in MCF-7 cells. (A) Cell apoptosis was determined following plasmid transduction. Data are expressed as the mean + standard deviation. ${ }^{* *} \mathrm{P}<0.01$, vs. control. (B) Western blot analysis of the protein expression levels of survivin and Bcl-2 using specific antibodies following plasmid tranduction. GAPDH was used as an internal control. GRIM-19, gene associated with retinoid-interferon-induced mortality 19; Bcl, B-cell lymphoma; pGRIM-19; GRIM-19-overexpression plasmid; pcDNA3.1, blank plasmid. 
A
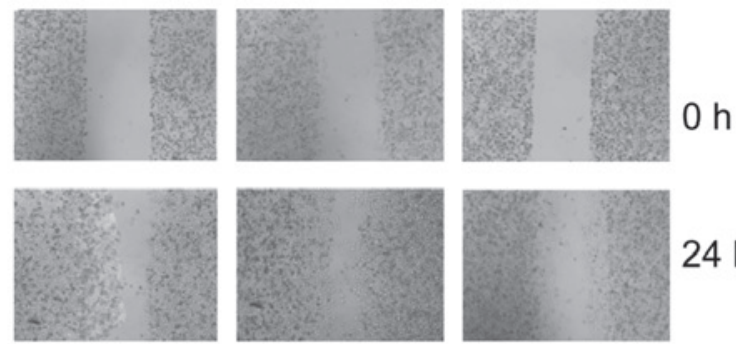

$24 \mathrm{~h}$

Control

pcDNA3.1 pGRIM-19
A

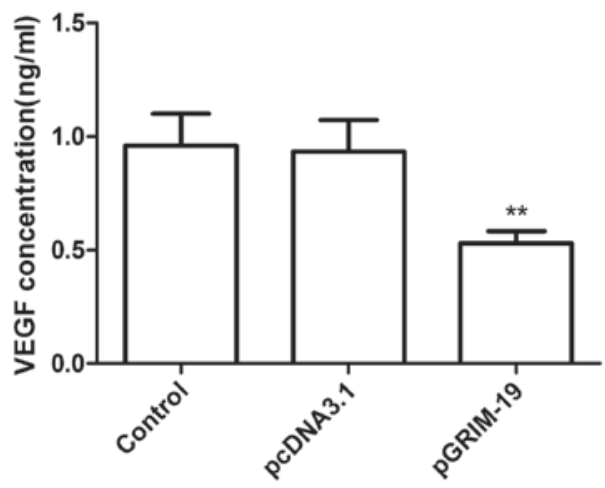

B

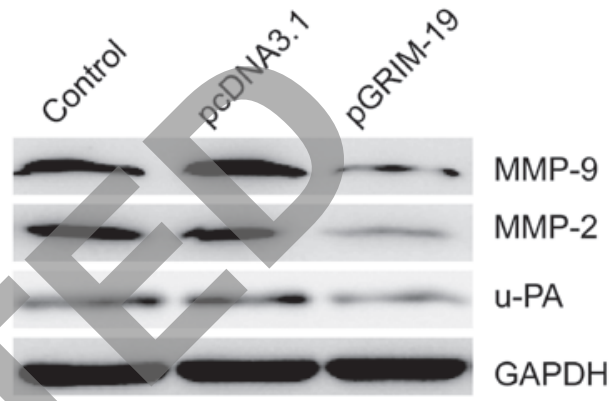

B

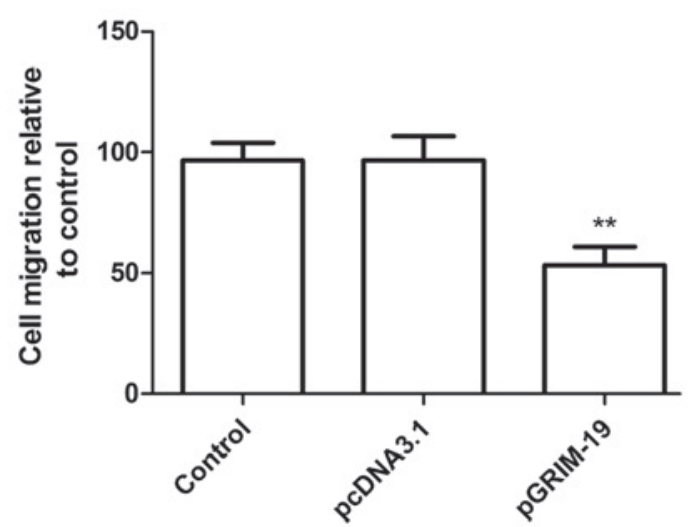

C

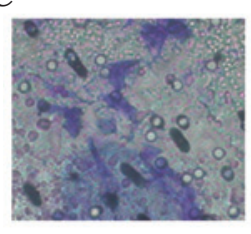

Control
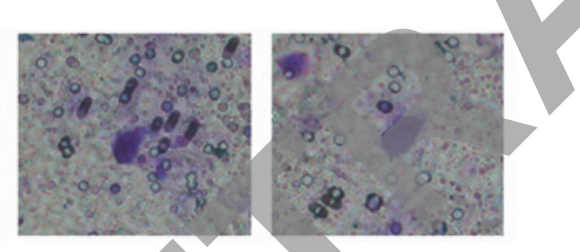

PGRIM-19
D

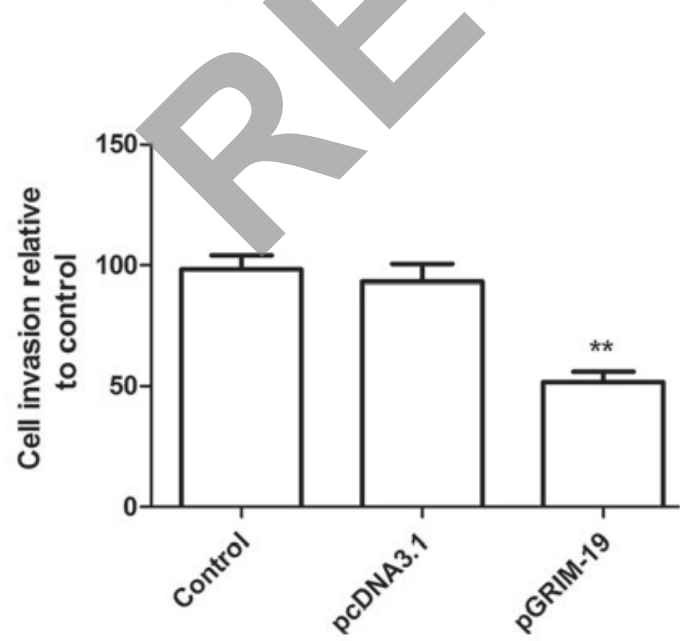

Figure 5. Upregulation of GRIM-19 inhibits the migration and invasion of MCF-7 cells. (A) Representative images of migration in MCF-7 cells transduced with the indicated plasmid (magnification, x20). (B) Determination of the numbers of migrating cells. (C) Representative images of the invasion of MCF-7 cells transduced with the indicated plasmid (magnification, x200). (D) Determination of the number of invading cells was counted. Data are expressed as the mean + standard deviation. ${ }^{* *} \mathrm{P}<0.01$, vs. control. GRIM-19, gene associated with retinoid-interferon-induced mortality 19; pGRIM-19, GRIM-19-overexpression plasmid; pcDNA3.1, blank plasmid. apoptosis, the apoptotic rate of the cells was assessed $48 \mathrm{~h}$ after treatment with pGRIM-19. Significantly higher levels of apoptosis were observed in the MCF-7 cells transfected with the pGRIM-19 plasmid, compared with the blank vector or control groups $(\mathrm{P}<0.05$; Fig. 4A).

Todetermine the potential mechanism of GRIM19-induced cell apoptosis in vitro, the protein expression levels of survivin and $\mathrm{Bcl}-2$ were determined using western blot analysis The results demonstrated that increased expression of GRIM-19 in the MCF-7 cells resulted in a notable decrease in the protein expression levels of survivin and $\mathrm{Bcl}-2$, compared with the blank vector or control groups $(\mathrm{P}<0.05$; Fig. 4B).

Upregulation of GRIM-19 inhibits migration and invasion in MCF-7 cells. To ascertain the inhibitory effect of the upregulation of GRIM-19 on breast cancer cell motility in vitro, a wound-healing assay was performed. As shown in Fig. 5A and B, the MCF-7 cells transfected with pGRIM-19 migrated significantly less than the untreated cells and the cells transfected with the blank vector $(\mathrm{P}<0.01)$

The ability of upregulated GRIM-19 to reduce the invasiveness of breast cancer cells was further investigated using a Transwell system assay. The invasion of the cells was also significantly decreased in the pGRIM-19 treatment group, compared with the control and the blank vector groups $(\mathrm{P}<0.01$; Fig. 5C and D). 
MMP-2, MMP-9, VEGF and $u-P A$ are involved in the invasiveness of breast cancer regulated by GRIM-19. The observation that GRIM-19 exerts an inhibitory effect on the migration and invasion of breast cancer cells, prompted examination of its effects on the expression levels of u-PA, MMP-2, MMP-9 and VEGF in breast cancer cells. As shown in Fig. 6A, ELISA analysis revealed that the level of VEGF excretion in the supernatant from the pGRIM-19-transfected cells was significantly decreased $(\mathrm{P}<0.01)$, compared with the blank vector and control groups. Western blot analysis revealed a marked decrease in the protein expression levels of MMP-2, MMP-9 and $\mathrm{u}-\mathrm{PA}$ in the supernatant and the total extracts from the pGRIM-19-transfected cells, compared with the blank vector and control groups (Fig. 6B). These findings suggested that the inhibitory effect of GRIM-19 on the invasion and metastasis of breast cancer was, at least partially, mediated by the downregulation of u-PA, MMP-9, MMP-2 and VEGF, which may contribute to degradation of the extracellular matrix (ETM).

\section{Discussion}

GRIM-19 was first identified as a novel cell death-regulatory gene, induced by a combination of IFN- $\beta$ and retinoic acid (5). It has been reported to be involved in numerous cellular functions, including apoptosis, proliferation, migration and invasion in various types of cancer (16-20). Several previous studies have demonstrated that introduction of the GRIM-19 gene into cancer cells using plasmid vectors inhibits tumor growth in vitro and in vivo (22-26). Zhang et al reported that overexpression of GRIM-19 in glioma cells using a transfection plasmid significantly suppressed cell proliferation and migration (24). Wang et al demonstrated that overexpression of GRIM-19 by transfection with the indicated plasmid suppresses the growth of lung adenocarcinoma in vitro and in vivo (25). Consistent with these results, the present study successfully constructed a eukaryotic vector expressing human GRIM-19, which exhibited highly efficient gene transduction in the MCF-7 cells. Using this plasmid, the induction of GRIM-19 in MCF-7 cells resulted in significant inhibition of cell proliferation, colony formation, migration and invasion, and induction of apoptosis. These findings are likely to contribute to the future development of GRIM-19-based gene therapeutic approaches for the treatment of breast cancer.

GRIM-19 was revealed as an inhibitor of STAT3 by binding to STAT3 and repressing its transcriptional activation (5). The downregulation of GRIM-19 is associated with increased activity of STAT3 in prostate cancer (27), cervical cancer (10), hepatocellular carcinoma (8) and breast cancer tissues (21). The STAT3 protein is reported to be important in carcinogenesis by promoting cell proliferation, differentiation and cell cycle progression, and inhibiting apoptosis $(17,28)$. Thus, the inhibition of STAT3 by GRIM-19 appears to be an important step in suppressing oncogenesis (29). Therefore, in the present study, a eukaryotic vector expressing human GRIM-19 (pGRIM-19) was constructed and transfected into MCF-7 cells. The resulting overexpression of GRIM-19 in the MCF-7 cells significantly inhibited the expression of STAT3, which further confirmed previous observations that GRIM-19 is an inhibitor of STAT3 (27,30).

Breast cancer is often lethal due to its aggressive metastasis, and migration and invasion are important features of cancer cells (31). Thus, the prevention of breast cancer recurrence and metastasis is of biological and clinical significance. ETM is a key stage in tumor invasion and metastasis, and is predominantly mediated by the secretion of MMP-2 (32), MMP-9 (33,34), VEGF (35), and the u-PA serine protease, (36). It has been demonstrated that aberrantly active STAT3 promotes tumor cell growth and survival via a continual induction of pro-growth genes, including VEGF $(35,37)$ and MMP-2 (32). GRIM-19, as a negative regulator of STAT3, had been observed to inhibit cell invasion and metastasis, and suppress the expression levels of VEGF, u-PA, MMP-9 and MMP-2 in several types of tumor $(18,23,26,37)$. Consistent with these results, the present study demonstrated that GRIM-19 inhibited the invasive and metastatic abilities of breast cancer cells and suppressed the expression levels of VEGF, u-PA, MMP-9 and MMP-2. Therefore, it was hypothesized that the inhibition of breast cancer cell invasion and migration by the pGRIM-19 plasmid is correlated with decreased expression levels of MMP-2, MMP-9, VEGF and $\mathrm{u}-\mathrm{PA}$, and is, at least in part, mediated by the STAT3 pathway

In conclusion, the present study provided evidence that the upregulation of GRIM-19 suppressed cell proliferation, colony formation, migration and invasion, and induced cell apoptosis in human breast cancer cells. Furthermore, overexpression of GRIM-19 in the MCF-7 cells suppressed invasion and migration by decreasing the expression levels of MMP-2, MMP-9, VEGF and u-PA, mediated by the STAT3 pathway. Collectively, these data suggested that upregulation of the GRIM-19 gene may be a potential approach to control the invasion and metastasis of human breast cancer.

\section{References}

1. Siegel R, Naishadham D and Jemal A: Cancer statistics, 2013. CA Cancer J Clin 63: 11-30, 2013.

2. DeSantis C, Ma J, Bryan L and Jemal A: Breast cancer statistics, 2013. CA Cancer J Clin 64: 52-62, 2014.

3. Murphy IG, Dillon MF, Doherty AO, et al: Analysis of patients with false negative mammography and symptomatic breast carcinoma. J Surg Oncol 96: 457-463, 2007.

4. Chen J and Wang X: MicroRNA-21 in breast cancer: diagnostic and prognostic potential. Clin Transl Oncol 16: 225-233, 2014.

5. Angell JE, Lindner DJ, Shapiro PS, Hofmann ER and Kalvakolanu DV: Identification of GRIM-19, a novel cell death-regulatory gene induced by the interferon-beta and retinoic acid combination, using a genetic approach. J Biol Chem 275: 33416-33426, 2000.

6. Fearnley IM, Carroll J, Shannon RJ, Runswick MJ, Walker JE and Hirst J: GRIM-19, a cell death regulatory gene product, is a subunit of bovine mitochondrial NADH:ubiquinone oxidoreductase (complex I). J Biol Chem 276: 38345-38348, 2001.

7. Huang G, Lu H, Hao A, et al: GRIM-19, a cell death regulatory protein, is essential for assembly and function of mitochondrial complex I. Mol Cell Biol 24: 8447-8456, 2004.

8. Li F, Ren W, Zhao Y, et al: Downregulation of GRIM-19 is associated with hyperactivation of p-STAT3 in hepatocellular carcinoma. Med Oncol 29: 3046-3054, 2012.

9. Alchanati I, Nallar SC, Sun P, et al: A proteomic analysis reveals the loss of expression of the cell death regulatory gene GRIM-19 in human renal cell carcinomas. Oncogene 25: 7138-7147, 2006.

10. Zhou Y, Li M, Wei Y, et al: Down-regulation of GRIM-19 expression is associated with hyperactivation of STAT3-induced gene expression and tumor growth in human cervical cancers. J Interferon Cytokine Res 29: 695-703, 2009.

11. Fan XY, Jiang ZF, Cai L and Liu RY: Expression and clinical significance of GRIM-19 in lung cancer. Med Oncol 29: 3183-3189, 2012 
12. Alchanati I, Nallar SC, Sun P, et al: A proteomic analysis reveals the loss of expression of the cell death regulatory gene GRIM-19 in human renal cell carcinomas. Oncogene 25: 7138-7147, 2006.

13. Wen LJ, Gao LF, Jin CS, et al: Small interfering RNA survivin and GRIM-19 co-expression salmonella plasmid inhibited the growth of laryngeal cancer cells in vitro and in vivo. Int J Clin Exp Pathol 6: 2071-2081, 2013.

14. Fusco A, Viglietto G and Santoro M: Point mutation in GRIM-19: a new genetic lesion in Hurthle cell thyroid carcinomas. Br J Cancer 92: 1817-1818, 2005.

15. Máximo V, Botelho T, Capela J, et al: Somatic and germline mutation in GRIM-19, a dual function gene involved in mitochondrial metabolism and cell death, is linked to mitochondrion-rich (Hurthle cell) tumours of the thyroid. Br J Cancer 92: 1892-1898, 2005.

16. Nallar SC, Kalakonda S, Lindner DJ, et al: Tumor-derived mutations in the gene associated with retinoid interferon-induced mortality (GRIM-19) disrupt its anti-signal transducer and activator of transcription 3 (STAT3) activity and promote oncogenesis. J Biol Chem 288: 7930-7941, 2013.

17. Lu Y, Fukuyama S, Yoshida R, et al: Loss of SOCS3 gene expression converts STAT3 function from anti-apoptotic to pro-apoptotic. J Biol Chem 281: 36683-36690, 2006

18. Huang Y, Yang M, Yang H and Zeng Z: Upregulation of the GRIM-19 gene suppresses invasion and metastasis of human gastric cancer SGC-7901 cell line. Exp Cell Res 316: 2061-2070, 2010.

19. Huang G, Chen Y, Lu H and Cao X: Coupling mitochondrial respiratory chain to cell death: an essential role of mitochondrial complex I in the interferon-beta and retinoic acid-induced cancer cell death. Cell Death Differ 14: 327-337, 2007.

20. Hao H, Liu J, Liu G, et al: Depletion of GRIM-19 accelerates hepatocellular carcinoma invasion via inducing EMT and loss of contact inhibition. J Cell Physiol 227: 1212-1219, 2012.

21. Zhou T, Chao L, Rong G, Wang C, Ma R and Wang X: Down-regulation of GRIM-19 is associated with STAT3 overexpression in breast carcinomas. Hum Pathol 44: 1773-1779, 2013.

22. Bu X, Zhao C, Wang W and Zhang N: GRIM-19 inhibits the STAT3 signaling pathway and sensitizes gastric cancer cells to radiation. Gene 512: 198-205, 2013.

23. Zhang L, Gao L,Li Y,etal: Effects of plasmid-based Stat3-specific short hairpin RNA and GRIM-19 on PC -3 M tumor cell growth Clin Cancer Res 14: 559-568, 2008.

24. Zhang Y, Hao H, Zhao S, et al: Downregulation of GRIM-19 promotes growth and migration of human glioma cells. Cancer Sci 102: 1991-1999, 2011.
25. Wang T, Yan XB, Zhao JJ, et al: Gene associated with retinoid-interferon-induced mortality-19 suppresses growth of lung adenocarcinoma tumor in vitro and in vivo. Lung Cancer 72 : 287-293, 2011.

26. Wang GM, Ren ZX, Wang PS, et al: Plasmid-based Stat3-specific siRNA and GRIM-19 inhibit the growth of thyroid cancer cells in vitro and in vivo. Oncol Rep 32: 573-580, 2014.

27. Zhang J, Yang J, Roy SK, et al: The cell death regulator GRIM-19 is an inhibitor of signal transducer and activator of transcription 3. Proc Natl Acad Sci USA 100: 9342-9347, 2003.

28. Darnell JE, Jr: STATs and gene regulation. Science 277: 1630-1635, 1997.

29. Kalakonda S, Nallar SC, Lindner DJ, Hu J, Reddy SP and Kalvakolanu DV: Tumor-suppressive activity of the cell death activator GRIM-19 on a constitutively active signal transducer and activator of transcription 3. Cancer Res 67: 6212-6220, 2007.

30. Lufei C, Ma J, Huang G, et al: GRIM-19, a death-regulatory gene product, suppresses Stat3 activity via functional interaction. EMBO J 22: 1325-1335, 2003.

31. Boyle ST and Kochetkova M: Breast cancer stem cells and the immune system: Promotion, evasion and therapy. J Mammary Gland Biol Neoplasia 19: 203-211, 2014.

32. Xie TX, Wei D, Liu M, et al: Stat3 activation regulates the expression of matrix metalloproteinase-2 and tumor invasion and metastasis. Oncogene 23: 3550-3560, 2004.

33. Farina AR, Tacconelli A, Vacca A, Maroder M, Gulino A and Mackay AR: Transcriptional up-regulation of matrix metalloproteinase-9 expression during spontaneous epithelial to neuroblast phenoty pe conversion by SK-N-SH neuroblastoma cells, involved in enhanced invasivity, depends upon GT-box and nuclear factor kappaB elements. Cell Growth Differ 10: 353-367, 1999.

34. Bond M, Fabunmi RP, Baker AH and Newby AC: Synergistic upregulation of metalloproteinase- 9 by growth factors and inflammatory cytokines: an absolute requirement for transcription factor NF-kappa B. FEBS Lett 435: 29-34, 1998.

35. Niu G, Wright KL, Huang M, et al: Constitutive Stat3 activity up-regulates VEGF expression and tumor angiogenesis. Oncogene 21: 2000-2008, 2002.

36. Cho JY, Chung HC, Noh SH, Roh JK, Min JS and Kim BS: High level of urokinase-type plasminogen activator is a new prognostic marker in patients with gastric carcinoma. Cancer 79: 878-883, 1997.

37. Wei D, Le X, Zheng L, et al: Stat3 activation regulates the expression of vascular endothelial growth factor and human pancreatic cancer angiogenesis and metastasis. Oncogene 22: 319-329, 2003. 